\title{
The effect of the introduction of NHS Direct on requests for telephone advice from an accident and emergency department
}

\author{
J Jones, M J Playforth
}

\begin{abstract}
Objectives-To assess the effect of the introduction of NHS Direct on advice seeking calls to an accident and emergency $(\mathrm{A} \& \mathrm{E})$ department.

Methods-Review of departmental telephone advice logbook before and after the introduction of NHS Direct together with recording of the number of calls redirected to NHS Direct by the hospital switchboard and the $A \& E$ department.

Results-The number of advice calls responded to within the department fell by $72.6 \%$ (84 in October 1998, 23 in October 1999). The number of calls redirected to NHS Direct was 242. The total number of calls to the hospital seeking advice thus increased by $315 \%$.

Conclusion-The introduction of NHS Direct has allowed for a mechanism to be put in place reducing the number of calls for advice being dealt with by the $A \& E$ department clinical staff with a concomitant time saving. At the same time the number of calls for advice made by the public to the hospital has dramatically increased.
\end{abstract}

(Emerg Med f 2001;18:300-301)

Keywords: NHS Direct; telephone advice

A number of calls for telephone advice are received each day by Pontefract Accident and Emergency department (district general hospital with approximately 4250 new attendances per month) and are dealt with by a doctor or, more commonly, a suitably experienced member of the nursing staff (D grade or above with at least six months accident and emergency (A\&E) experience). Before April 1999 all calls to the hospital requesting general health advice were directed to A\&E. It has been departmental policy to keep a written log of the advice given and the time taken to give it for many years. An earlier audit of 817 calls to the department (over seven months, giving an average of 117 per month) suggested that the average call duration was 3.8 minutes. ${ }^{1}$ In April 1999 NHS Direct was introduced to the area covered by this hospital. Callers to the service speak to specially trained qualified nurses using a computer based protocol system to provide appropriate advice including from whom to seek further assistance (GP, A\&E, pharmacist, etc). The NHS Direct service is also linked to the local ambulance control allowing the NHS Direct staff to directly request an ambulance for the caller. With the introduction of this service a system was put in place at our hospital whereby the NHS Direct telephone number is given to callers to the hospital seeking general health advice by the switchboard staff (unless they specifically ask to be put through to $A \& E$ ). Calls via a direct line to $\mathrm{A} \& \mathrm{E}$ reception are similarly dealt with. The NHS Direct number has also been widely advertised in the media and every household in the area has received a leaflet outlining the NHS Direct service and how to access it. Some calls may still reach clinical staff, either by a direct line to the nursing station or from reception or switchboard at the caller's request. We wished to assess the impact of the introduction of NHS Direct, together with our mechanism for the redirection of calls, on the number of calls requesting health advice reaching clinical staff.

\section{Method}

The telephone advice logbook was reviewed to assess the number of calls for advice dealt with by the department for the months of October 1998 and October 1999. A prospective record was kept by switchboard and by the A\&E Reception staff of callers redirected to NHS Direct during October 1999. The monthly new attendance figures for the department were recorded (as is usual policy).

\section{Results}

The number of calls requesting health advice dealt with by A\&E clinical staff fell from 84 in October 1998 to 23 in October 1999 (a 72.6\% reduction). A further 242 calls were referred to NHS Direct in October 1999 (116 by A\&E reception and 126 by the hospital switchboard). The hospital as a whole thus received 265 calls for advice in October 1999, an increase of $315 \%$ from the previous year. There were 4055 new attendances in October 1998 and 4268 in October 1999, an increase of $5.2 \%$.

\section{Discussion}

Before discussing the results and their possible implications a potential methodological flaw needs to be outlined. All calls requesting health advice made to the hospital in 1998 should have been transferred to $\mathrm{A} \& \mathrm{E}$ and thus recorded in the logbook. Experience suggests that on occasion not all calls are recorded, particularly during busy periods. During October 1999 written reminders were distributed to staff to encourage logging of calls so if anything 
the proportion of calls recorded should have increased, increasing the validity of the recorded drop in calls dealt with by A\&E clinical staff. As a new, and potentially more reliable mechanism for recording the number of calls to the hospital in October 1999 was instituted it is possible, if a substantial proportion of calls went unrecorded, that the calculated $315 \%$ increase described above is an overestimate. However, as the importance of recording advice given is heavily emphasised within the department (primarily for medicolegal reasons) it is hoped that the proportion is only small.

The provision of telephone advice is a potentially time consuming exercise. Fatovich et al reviewing 1682 calls for telephone advice found a mean call duration of 3.9 minutes, ${ }^{1}$ a figure very much in line with our own audit results of 3.8 minutes (unpublished data). This is equivalent to nearly 5.5 hours of staff time in our department in October 1998 decreasing to less than two hours in October 1999. Crouch et al suggest an average number of calls for $\mathrm{A} \& \mathrm{E}$ and minor injuries units in the UK is 15.5 per weekday and 21.0 per weekend day (compared with 2.5 per weekday and 3.1 per weekend day for this hospital before the introduction of NHS Direct). ${ }^{2}$ They extrapolate that nearly 2 million calls are dealt with in the UK each year. A $72.6 \%$ reduction in calls throughout the UK could therefore produce a time saving of approximately 90000 hours per year. But the introduction of NHS Direct together with its attendant publicity, while allowing the reduction in the number of calls dealt with by our department, seems to have stimulated (or simply met) the demand for information from the public. From the extrapolation by Crouch et al a national $300 \%$ increase in calls for telephone advice would require an extra 390000 hours of someone's time (roughly equivalent to 230 full time nurses) and the observed $300 \%$ increase in calls to our hospital is in addition to those calls going directly to NHS Direct. A further factor is the addition to the workload of the ancillary staff within the hospital who have to redirect the calls. Even if this takes only $30 \mathrm{sec}-$ onds this would still consume two hours of their time each month in this hospital alone, although this would be expected to fall with increasing public familiarity with the NHS Direct service. Although there has been a 5.2\% increase in new attendances when comparing October 1998 to October 1999 it is not possible to draw any significant conclusions about the effect of NHS Direct on actual A\&E attendances from these isolated figures. A recent observational study suggests that the initial introduction of NHS Direct had minimal impact on the overall demand for immediate care services and comments that the calls made to the service seem to be simply an addition to the overall picture of health service use. ${ }^{3}$

Contributors

Michael Playforth initiated the study and contributed to the writing of the paper. Jonathan Jones designed the study, collected and analysed data, wrote the paper and acts as guarantor.

Funding: none.

Conflicts of interest: none.

1 Fatovich DM, Jacobs IG, McCance JP, et al. Emergency department telephone advice. Med f Aust 1998;169:143-6. 2 Crouch R, Dale J, Visavadia B, et al. Provision of telephone advice from accident and emergency departments: a national survey. F Accid Emerg Med 1999;16:112-13.

3 Munro J, Nicholl J, O'Cathain A, et al. Impact of NHS Direct on demand for immediate care: observational study. BMF 2000;321:150-3. 